\title{
Existence and uniqueness of solutions for multi-term fractional $q$-integro-differential equations via quantum calculus
}

\author{
Sotiris K. Ntouyas ${ }^{1,2}$ (1) and Mohammad Esmael Samei ${ }^{3^{*}}$ (B)
}

\section{"Correspondence:}

mesamei@gmail.com;

mesamei@basu.ac.ir

${ }^{3}$ Department of Mathematics,

Faculty of Basic Science, Bu-Ali Sina University, Hamedan, Iran

Full list of author information is available at the end of the article

\section{严 Springer}

\begin{abstract}
In this investigation, by applying the definition of the fractional $q$-derivative of the Caputo type and the fractional q-integral of the Riemann-Liouville type, we study the existence and uniqueness of solutions for a multi-term nonlinear fractional $q$-integro-differential equations under some boundary conditions ${ }^{c} D_{q}^{\alpha} x(t)=w\left(t, x(t),\left(\varphi_{1} x\right)(t),\left(\varphi_{2} x\right)(t),{ }^{c} D_{q}^{\beta_{1}} x(t),{ }^{c} D_{q}^{\beta_{2}} x(t), \ldots,{ }^{C} D_{q}^{\beta_{n}} x(t)\right)$. Our results are based on some classical fixed point techniques, as Schauder's fixed point theorem and Banach contraction mapping principle. Besides, some instances are exhibited to illustrate our results and we report all algorithms required along with the numerical result obtained.
\end{abstract}

MSC: Primary 34A08; 39A13; secondary 34K37

Keywords: Multi-term fractional q-integro-differential equation; Caputo q-derivative; Quantum calculus; Fixed point

\section{Introduction}

The subjects of fractional calculus and $q$-calculus are one of the significant branches in mathematical analysis. In 1910, the subject of $q$-difference equations was introduced by Jackson [1]. After that, at the beginning of the last century, studies on the $q$-difference equation appeared in much work, especially in [2-6]. For some earlier work on the topic, we refer to $[7,8]$, whereas the preliminary concepts on $q$-fractional calculus can be found in [9], as indicated: Perhaps Leibniz did not expect this number of applications when he sent a letter in 1695 to L'Hopital asking about the meaning of the derivative of order half. For countless applications on the $q$-fractional calculus, see for example [10-17].

In the recent years, there have appeared many papers about differential and integrodifferential equations and inclusions which are valuable tools in the modeling of many phenomena in various fields of science [18-25]. In 2012, Ahmad et al. [26] discussed the existence and uniqueness of solutions for the fractional $q$-difference equations ${ }^{c} D_{q}^{\alpha} u(t)=$ $T(t, u(t)), \alpha_{1} u(0)-\beta_{1} D_{q} u(0)=\gamma_{1} u\left(\eta_{1}\right)$ and $\alpha_{2} u(1)-\beta_{2} D_{q} u(1)=\gamma_{2} u\left(\eta_{2}\right)$, for $t \in I$, where $\alpha \in(1,2], \alpha_{i}, \beta_{i}, \gamma_{i}, \eta_{i}$ are real numbers, for $i=1,2$ and $T \in C(J \times \mathbb{R}, \mathbb{R})$. In 2013, Zhao $e l$ al. [27] reviewed the $q$-integral problem $\left(D_{q}^{\alpha} u\right)(t)+f(t, u(t))=0$, with the conditions that $u(1), u(0)$ are equal to $\mu I_{q}^{\beta} u(\eta), 0$, respectively, for almost all $t \in(0,1)$, where $q \in(0,1)$ and $\alpha, \beta, \eta$ belong to $(1,2],(0,2],(0,1)$, respectively, $\mu$ is positive real number, $D_{q}^{\alpha}$ is the

(c) The Author(s) 2019. This article is distributed under the terms of the Creative Commons Attribution 4.0 International License (http://creativecommons.org/licenses/by/4.0/), which permits unrestricted use, distribution, and reproduction in any medium, provided you give appropriate credit to the original author(s) and the source, provide a link to the Creative Commons license, and indicate if changes were made. 
$q$-derivative of Riemann-Liouville and we have a real-valued continuous map $u$ defined on $I \times[0, \infty)$. In 2014, Ahmad et al. [28] considered the problem

$$
\left\{\begin{array}{l}
{ }^{c} D_{q}^{\beta}\left({ }^{c} D_{q}^{\gamma}+\lambda\right) u(t)=p f(t, u(t))+k I_{q}^{\xi} g(t, u(t)), \\
\alpha_{1} u(0)-\left.\beta_{1}\left(t^{(1-\gamma)} D_{q} u(0)\right)\right|_{t=0}=\sigma_{1} u\left(\eta_{1}\right), \quad \alpha_{2} u(1)+\beta_{2} D_{q} u(1)=\sigma_{2} u\left(\eta_{2}\right),
\end{array}\right.
$$

for $t, q \in[0,1]$, where ${ }^{c} D_{q}^{\beta}$ and ${ }^{c} D_{q}^{\gamma}$ denote the fractional $q$-derivative of the Caputo type, $0<\beta, \gamma \leq 1, I_{q}^{\xi}($.) denotes the Riemann-Liouville integral with $\xi \in(0,1), f, g$ are given continuous functions, $\lambda$ and $p, k$ are real constants and $\alpha_{i}, \beta_{i}, \sigma_{i} \in \mathbb{R}, \eta_{i} \in(0,1), i=1,2$. Also, one may refer to some research of Ahmad et al., in the recent years in [12, 14, 2931]. In 2016, Abdeljawad et al. [32] stated and proved a new discrete $q$-fractional version of Gronwall inequality, ${ }_{q} C_{a}^{\alpha} u(t)=T(t, u(t))$, where $u(a)=\gamma$, such that $\alpha \in(0,1], a \in \mathbb{T}_{q}=\left\{q^{n}\right.$ : $n \in \mathbb{Z}\}$, t belongs to $\mathbb{T}_{a}=[0, \infty)_{q}=\left\{q^{-i} a: i=0,1,2, \ldots\right\},{ }_{q} C_{a}^{\alpha}$ means the Caputo fractional difference of order $\alpha$ and $T(t, x)$ fulfills a Lipschitz condition for all $t$ and $x$. In 2019, Samei et al. [25] investigated the existence of solutions for equations and inclusions of multi-term fractional $q$-integro-differential with non-separated and initial boundary conditions

In this article, motivated by these achievements and the following results, we are working to stretch out solutions for the multi-term nonlinear fractional $q$-integro-differential equation with boundary conditions,

$$
{ }^{c} D_{q}^{\alpha} x(t)=w\left(t, x(t),\left(\varphi_{1} x\right)(t),\left(\varphi_{2} x\right)(t),{ }^{c} D_{q}^{\beta_{1}} x(t),{ }^{c} D_{q}^{\beta_{2}} x(t), \ldots,{ }^{c} D_{q}^{\beta_{n}} x(t)\right),
$$

under conditions $x(0)+a x(1)=0$ and $x^{\prime}(0)+b x^{\prime}(1)=0$, for $t \in J:=[0,1]$ and all $q \in(0,1)$, where $1<\alpha<2, \beta_{i} \in(0,1)$ with $i=1,2, \ldots, n, a, b \neq-1, w: J \times \mathbb{R}^{n+3} \rightarrow \mathbb{R}$ is continuous for all variables and the mappings $\gamma_{j}$ map $J \times J$ to $\mathbb{R}^{+}$such that $\sup _{t \in J} \mid \int_{0}^{t} \gamma_{j}(t, s) d_{q} s$, where $j=1,2$, are finite, the maps $\varphi_{j}$, where $j=1,2$, are defined by $\left(\varphi_{j} u\right)(t)=\int_{0}^{t} \gamma_{j}(t, s) u(s) d_{q} s$.

The rest of the paper is arranged as follows: in Sect. 2, we recall some preliminary concepts and fundamental results of $q$-calculus. Section 3 is devoted to the main results, while examples illustrating the obtained results and algorithm for the problems are presented in Sect. 4.

\section{Preliminaries}

First of all, we point out some of the materials on the fractional $q$-calculus and fundamental results of it which needed in the next sections (for more information, consider [1, 9-11, 33]). Then, some well-known theorems of fixed point theorems are presented.

Assume that $q \in(0,1)$ and $a \in \mathbb{R}$. Define $[a]_{q}=\frac{1-q^{a}}{1-q}[1]$. The power function $(x-y)_{q}^{n}$ with $n \in \mathbb{N}_{0}$ is $(x-y)_{q}^{(n)}=\prod_{k=0}^{n-1}\left(x-y q^{k}\right)$ and $(x-y)_{q}^{(0)}=1$ where $x, y \in \mathbb{R}$ and $\mathbb{N}_{0}:=\{0\} \cup \mathbb{N}$ [10]. Also, for $\alpha \in \mathbb{R}$ and $a \neq 0$, we have $(x-y)_{q}^{(\alpha)}=x^{\alpha} \prod_{k=0}^{\infty}\left(x-y q^{k}\right) /\left(x-y q^{\alpha+k}\right)$. If $y=0$, then it is clear that $x^{(\alpha)}=x^{\alpha}$ (Algorithm 1). The $q$-Gamma function is given by $\Gamma_{q}(z)=$ $(1-q)^{(z-1)} /(1-q)^{z-1}$, where $z \in \mathbb{R} \backslash\{0,-1,-2, \ldots\}[1]$. Note that $\Gamma_{q}(z+1)=[z]_{q} \Gamma_{q}(z)$. The value of the $q$-Gamma function, $\Gamma_{q}(z)$, for input values $q$ and $z$ with counting the number of sentences $n$ in summation is addressed by a simplifying analysis. For this design, we present a pseudo-code description of the technique for estimating $q$-Gamma function of order $n$ which show in Algorithm 2. The $q$-derivative of the function $f$ is defined by $\left(D_{q} f\right)(x)=\frac{f(x)-f(q x)}{(1-q) x}$ and $\left(D_{q} f\right)(0)=\lim _{x \rightarrow 0}\left(D_{q} f\right)(x)$, which is shown in Algorithm 3 [4]. Also, 

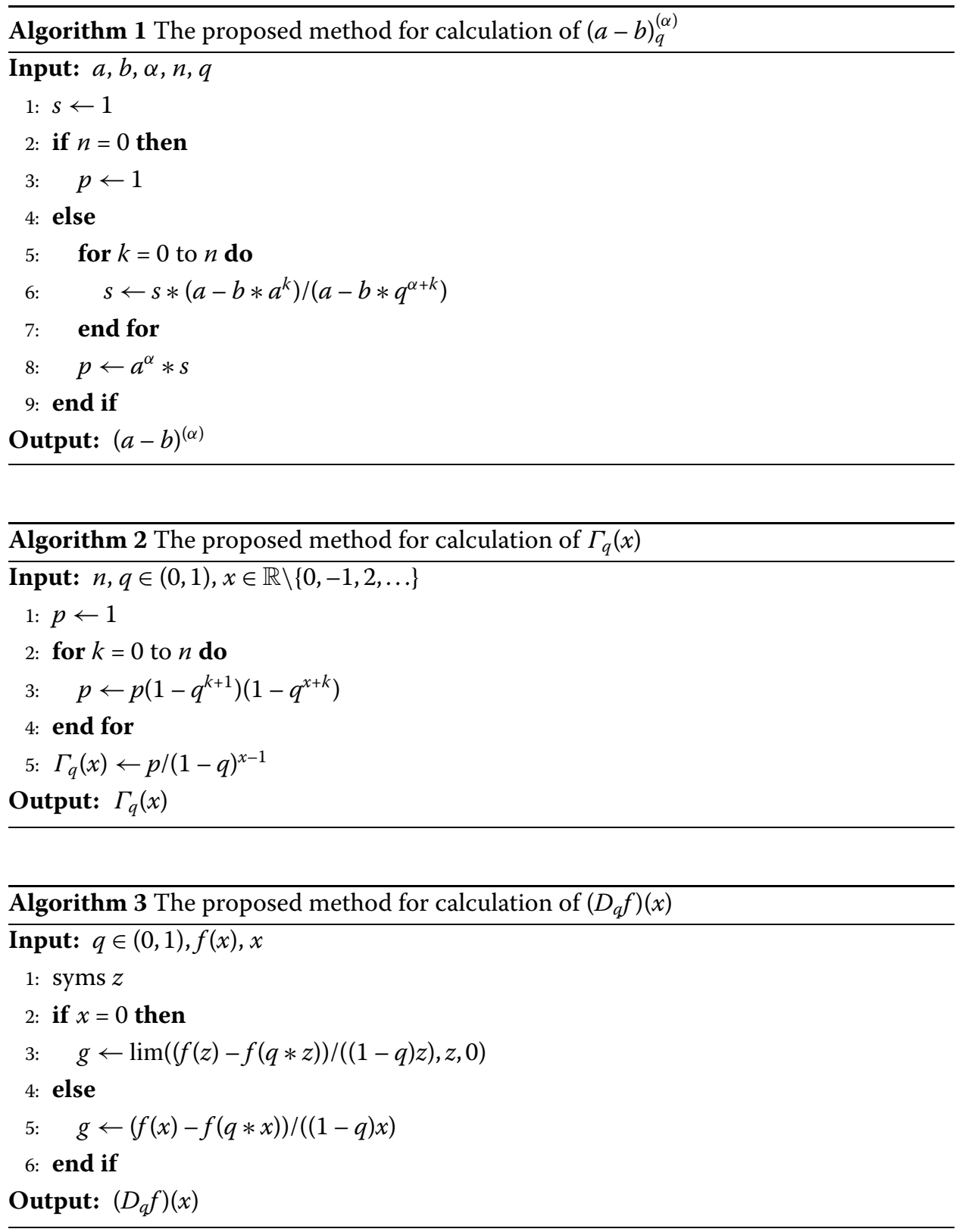

the higher order $q$-derivative of a function $f$ is defined by $\left(D_{q}^{n} f\right)(x)=D_{q}\left(D_{q}^{n-1} f\right)(x)$ for all $n \geq 1$, where $\left(D_{q}^{0} f\right)(x)=f(x)$ [4]. The $q$-integral of a function $f$ defined on $[0, b]$ is defined by

$$
I_{q} f(x)=\int_{0}^{x} f(s) d_{q} s=x(1-q) \sum_{k=0}^{\infty} q^{k} f\left(x q^{k}\right)
$$

for $0 \leq x \leq b$, provided that the series absolutely converges [4]. For any positive number $\alpha$ and $\beta$, the $q$-Beta function is defined by [33]

$$
B_{q}(\alpha, \beta)=\int_{0}^{1}(1-q s)_{q}^{(\alpha-1)} s^{\beta-1} d_{q} s
$$




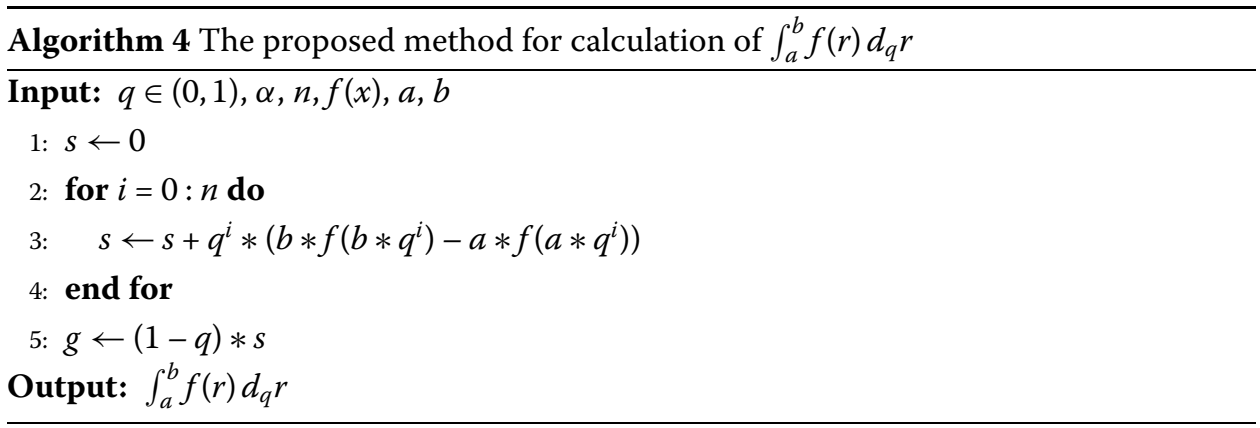

The $q$-derivative of the function $f$ is defined by $\left(D_{q} f\right)(x)=\frac{f(x)-f(q x)}{(1-q) x}$ and $\left(D_{q} f\right)(0)=$ $\lim _{x \rightarrow 0}\left(D_{q} f\right)(x)$, which is shown in Algorithm $3[4,11,33]$. If $a$ is in $[0, b]$, then

$$
\int_{a}^{b} f(u) d_{q} u=I_{q} f(b)-I_{q} f(a)=(1-q) \sum_{k=0}^{\infty} q^{k}\left[b f\left(b q^{k}\right)-a f\left(a q^{k}\right)\right]
$$

whenever the series exists, which is shown in Algorithm 4. The operator $I_{q}^{n}$ is given by $\left(I_{q}^{0} h\right)(x)=h(x)$ and

$$
\left(I_{q}^{n} h\right)(x)=\left(I_{q}\left(I_{q}^{n-1} h\right)\right)(x)
$$

for $n \geq 1$ and $g \in C([0, b])[4]$. It has been proved that $\left(D_{q}\left(I_{q} f\right)\right)(x)=f(x)$ and $\left(I_{q}\left(D_{q} f\right)\right)(x)=$ $f(x)-f(0)$ whenever $f$ is continuous at $x=0$ [4]. The fractional Riemann-Liouville type $q$-integral of the function $f$ on $J$, of $\alpha \geq 0$ is given by $\left(I_{q}^{0} f\right)(t)=f(t)$ and

$$
\left(I_{q}^{\alpha} f\right)(t)=\frac{1}{\Gamma_{q}(\alpha)} \int_{0}^{t}(t-q s)^{(\alpha-1)} f(s) d_{q} s,
$$

for $t \in J$ and $\alpha>0[31,34]$. Also, the fractional Caputo type $q$-derivative of the function $f$ is given by

$$
\begin{aligned}
\left({ }^{c} D_{q}^{\alpha} f\right)(t) & =\left(I_{q}^{[\alpha]-\alpha}\left(D_{q}^{[\alpha]} f\right)\right)(t) \\
& =\frac{1}{\Gamma_{q}([\alpha]-\alpha)} \int_{0}^{t}(t-q s)^{([\alpha]-\alpha-1)}\left(D_{q}^{[\alpha]} f\right)(s) d_{q} s,
\end{aligned}
$$

for $t \in J$ and $\alpha>0$ [31, 34]. It has been proved that $\left(I_{q}^{\beta}\left(I_{q}^{\alpha} f\right)\right)(x)=\left(I_{q}^{\alpha+\beta} f\right)(x)$ and $\left(D_{q}^{\alpha}\left(I_{q}^{\alpha} f\right)\right)(x)=f(x)$, where $\alpha, \beta \geq 0$ [34]. By using Algorithm 2, we can calculate $\left(I_{q}^{\alpha} f\right)(x)$ which is shown in Algorithm 5.

Theorem 1 (Schauder's fixed point theorem [35]) Let E be a closed, convex and bounded subset of a Banach space $X$ and self-map $T$ defined on $E$ be continuous. Then $T$ has a fixed point in $E$ whenever $T(E)$ is a relatively compact subset of $X$.

\section{Main results}

Here, we investigate the inclusion of fractional $q$-derivative (1). First, we recall the following key result. 


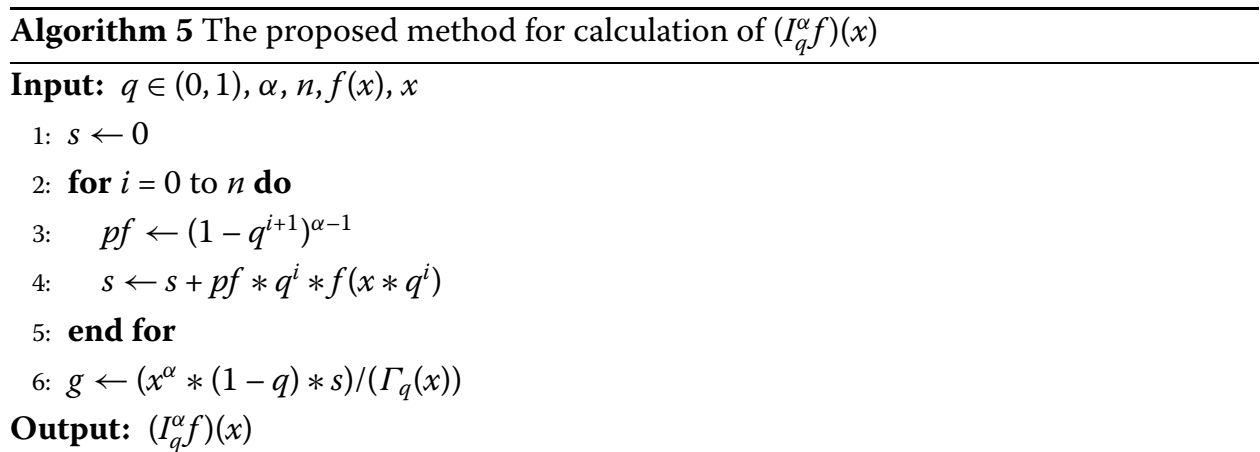

Lemma 2 ([17]) Let $\alpha>0$ and $n=[\alpha]+1$. Then $I_{q}^{\alpha c} D_{q}^{\alpha} x(t)=x(t)+c_{0}+c_{1} t+c_{2} t+\cdots+$ $c_{n-1} t^{n-1}$, where $c_{0}, c_{1}, \ldots, c_{n-1}$ belong to $\mathbb{R}$.

Let us define the set $X$ of all $f \in C(I)$, such that ${ }^{c} D_{q}^{\beta_{i}} x$ belongs to $C(I)(i=1,2, \ldots, n)$ and $q \in(0,1)$, where $0<\beta_{i}<1$. It is known that $(X,\|\cdot\|)$ with the norm $\|x\|=\max _{t \in J}|x(t)|+$ $\sum_{i=1}^{n} \max _{t \in J}\left|{ }^{c} D_{q}^{\beta_{i}} x(t)\right|$, is a Banach space.

Lemma 3 Suppose that $f$ in $C(J)$ and $\alpha \in(1,2)$. Then the boundary value problem

$$
\left\{\begin{array}{l}
{ }^{c} D_{q}^{\alpha} x(t)=f(t), \quad t \in J, \\
x(0)+a x(1)=0, \quad x^{\prime}(0)+b x^{\prime}(1)=0,
\end{array}\right.
$$

is equivalent to the following q-integral equation:

$$
x(t)=I_{q}^{\alpha} f(t)-I_{q}^{\alpha} f(1)+\frac{a b-b(1+a) t}{(1+a)(1+b)} I_{q}^{\alpha-1} f(1) .
$$

Proof First of all, we see that Lemma 2 implies that

$$
x(t)=\int_{0}^{t} \frac{(t-q s)^{(\alpha-1)}}{\Gamma_{q}(\alpha)} f(s) d_{q} s+c_{1} t+c_{2},
$$

where $c_{1}, c_{2}$ are arbitrary constants. By applying the boundary conditions we find

$$
\begin{aligned}
& c_{1}=-\frac{b}{1+b} I_{q}^{\alpha-1} f(1), \\
& c_{2}=-\frac{a}{1+a} I_{q}^{\alpha} f(1)+\frac{a b}{(1+a)(1+b)} I_{q}^{\alpha-1} f(1) .
\end{aligned}
$$

Substituting $c_{1}$ and $c_{2}$ in (5) we get (4). The converse follows by direct computation. The proof is completed.

Theorem 4 Let $\ell \in L^{\frac{1}{\kappa}}\left(J, \mathbb{R}^{+}\right), 0<\kappa<\alpha-1$ such that

$$
\left|F_{t, x_{i}, u_{i}}-F_{t, x_{i}^{\prime}, v_{i}}\right| \leq \ell(t)\left(\sum_{i=1}^{3}\left|x_{i}-x_{i}^{\prime}\right|+\sum_{i=1}^{n}\left|u_{i}-v_{i}\right|\right),
$$

for each $t \in J, x_{i}, x_{i}^{\prime}$, with $i=1,2,3$ and $u_{1}, u_{2}, \ldots, u_{n}, v_{1}, v_{2}, \ldots, v_{n} \in \mathbb{R}$, where $F_{t, x_{i}, u_{i}}=$ $w\left(t, x_{1}, x_{2}, x_{3}, u_{1}, u_{2}, \ldots, u_{n}\right)$ and $F_{t, x_{i}^{\prime} v_{i}}=w\left(t, x_{1}^{\prime}, x_{2}^{\prime}, x_{3}^{\prime}, v_{1}, v_{2}, \ldots, v_{n}\right)$. Then the problem (1) 
has a unique solution provided

$$
\begin{aligned}
\Delta= & \left(1+{ }_{0} \lambda_{1}+{ }_{0} \lambda_{2}\right)\left[\frac{(1+2 a) \ell^{*} k_{1}}{(1+a) \Gamma_{q}(\alpha)}+\frac{b \ell^{*} k_{2}}{(1+a)(1+b) \Gamma_{q}(\alpha-1)}\right. \\
& \left.+\sum_{i=1}^{n}\left(\frac{\Gamma_{q}(\alpha-\kappa) \ell^{*} k_{2}}{\Gamma_{q}(\alpha-1) \Gamma_{q}\left(\alpha-\beta_{i}-\kappa+1\right)}+\frac{b \ell^{*} k_{2}}{(1+b) \Gamma_{q}\left(2-\beta_{i}\right) \Gamma_{q}(\alpha-1)}\right)\right] \\
<1 &
\end{aligned}
$$

where

$$
{ }_{0} \lambda_{i}=\sup _{t \in J}\left|\int_{0}^{t} \gamma_{i}(t, s) d_{q} s\right|, \quad i=1,2, \quad \ell^{*}=\left(\int_{0}^{1}(\ell(s))^{\frac{1}{\kappa}} d_{q} s\right)^{\kappa},
$$

$k_{1}=\left(\frac{1-\kappa}{\alpha-\kappa}\right)^{1-\kappa}$ and $k_{2}=\left(\frac{1-\kappa}{\alpha-\kappa-1}\right)^{1-\kappa}$.

Proof Briefly, we put

$$
F_{u(s)}=w\left(s, u(s),\left(\varphi_{1} u\right)(s),\left(\varphi_{2} u\right)(s),{ }^{c} D_{q}^{\beta_{1}} u(s),{ }^{c} D_{q}^{\beta_{2}} u(s), \ldots,{ }^{c} D_{q}^{\beta_{n}} u(s)\right),
$$

and using Lemma 3, we define a self-map $T$ on $X$ by

$$
(T u)(t)=I_{q} \alpha F_{u(t)}-I_{q}^{\alpha} F_{u(1)}+g(t) I_{q}^{\alpha-1} F_{u(1)}
$$

where $a_{0}=\frac{a}{1+a}$ and $g(t)=\frac{a b-b(1+a) t}{(1+a)(1+b)}$ is a real-valued function on $J$. At present, by using the Hölder inequality, for each $u, v \in X$ and $t \in J$, we get

$$
\begin{aligned}
|(T u)(t)-(T v)(t)|= & \mid I_{q}^{\alpha}\left(F_{u(t)}-F_{v(t)}\right)-a_{0} I_{q}^{\alpha}\left(F_{u(1)}-F_{v(1)}\right) \\
& +g(t) I_{q}^{\alpha-1}\left(F_{u(1)}-F_{v(1)}\right) \mid \\
\leq & I_{q}^{\alpha}\left|F_{u(t)}-F_{v(t)}\right|+a_{1} I_{q}^{\alpha}\left|F_{u(1)}-F_{v(1)}\right| \\
& +|g(t)| I_{q}^{\alpha-1}\left|F_{u(1)}-F_{v(1)}\right| \\
\leq & I_{q}^{\alpha}\left(\ell ( t ) \left(|u(t)-v(t)|+\sum_{i=1}^{2}\left|\left(\varphi_{i} u\right)(t)-\left(\varphi_{i} v\right)(t)\right|\right.\right. \\
& \left.\left.+\sum_{i=1}^{n}\left|{ }^{c} D_{q}^{\beta_{i}} u(t)-{ }^{c} D_{q}^{\beta_{i}} v(t)\right|\right)\right) \\
& +a_{1} I_{q}^{\alpha}(\ell(1)(|u(1)-v(1)| \\
& +\sum_{i=1}^{2}\left|\left(\varphi_{i} u\right)(1)-\left(\varphi_{i} v\right)(1)\right| \\
& \left.\left.+\sum_{i=1}^{n}\left|{ }^{c} D_{q}^{\beta_{i}} u(1)-{ }^{c} D_{q}^{\beta_{i}} v(1)\right|\right)\right) \\
& +a_{2}(1+2|a|) I_{q}^{\alpha-1}(\ell(1)(|u(1)-v(1)|
\end{aligned}
$$




$$
\begin{aligned}
& +\sum_{i=1}^{2}\left|\left(\varphi_{i} u\right)(1)-\left(\varphi_{i} v\right)(1)\right| \\
& \left.\left.+\sum_{i=1}^{n}\left|{ }^{c} D_{q}^{\beta_{i}} u(1)-{ }^{c} D_{q}^{\beta_{i}} v(1)\right|\right)\right) \\
& \leq b_{1} d \int_{0}^{t}(t-q s)^{(\alpha-1)} \ell(s) d_{q} s \\
& +a_{1} b_{1} d \int_{0}^{1}(1-q s)^{(\alpha-1)} \ell(s) d_{q} s \\
& +a_{2} b_{2}(1+2|a|) d \int_{0}^{1}(1-q s)^{(\alpha-2)} \ell(s) d_{q} s \\
& \leq b_{1} d\left(\int_{0}^{t}\left((t-q s)^{(\alpha-1)}\right)^{\frac{1}{1-\kappa}} d_{q} s\right)^{1-\kappa}\left(\int_{0}^{t}(\ell(s))^{\frac{1}{\kappa}} d_{q} s\right)^{\kappa} \\
& +a_{1} b_{1} d\left(\int_{0}^{1}\left((1-q s)^{(\alpha-1)}\right)^{\frac{1}{1-\kappa}} d_{q} s\right)^{1-\kappa} \\
& \times\left(\int_{0}^{1}(\ell(s))^{\frac{1}{\kappa}} d_{q} s\right)^{\kappa} \\
& +a_{2} b_{2}(1+2|a|) d\left(\int_{0}^{1}\left((1-q s)^{(\alpha-2)}\right)^{\frac{1}{1-\kappa}} d_{q} s\right)^{1-\kappa} \\
& \times\left(\int_{0}^{1}(\ell(s))^{\frac{1}{\kappa}} d_{q} s\right)^{\kappa} \\
& \leq b_{1} \ell^{*} d k_{1}+a_{1} b_{1} \ell^{*} d k_{1}+a_{2} b_{2}(1+2|a|) \ell^{*} d k_{2} \\
& \leq\left[\frac{(1+2|a|) b_{1} \ell^{*}}{|1+a|} k_{1}+a_{2}(1+2|a|) b_{2} \ell^{*} k_{2}\right] d,
\end{aligned}
$$

where $d=\|u-v\|, a_{1}=\frac{|a|}{|1+a|}, a_{2}=\frac{|b|}{|1+a||1+b|}, b_{1}=\frac{1+0 \lambda_{1}+0 \lambda_{2}}{\Gamma_{q}(\alpha)}$ and $b_{2}=\frac{1+0 \lambda_{1}+0 \lambda_{2}}{\Gamma_{q}(\alpha-1)}$. Also, we have

$$
\begin{aligned}
\left|{ }^{c} D_{q}^{\beta_{i}}(T u)(t)-{ }^{c} D_{q}^{\beta_{i}}(T v)(t)\right|= & \left|I_{q}^{1-\beta_{i}}(T u)^{\prime}(t)-I_{q}^{1-\beta_{i}}(T v)^{\prime}(t)\right| \\
= & \mid I_{q}^{1-\beta_{i}}\left(I_{q}^{\alpha-1} F_{u(s)}-\frac{b}{1+b} I_{q}^{\alpha-1} F_{u(1)}\right) \\
& -I_{q}^{1-\beta_{i}}\left(I_{q}^{\alpha-1} F_{\nu(s)}-\frac{b}{1+b} I_{q}^{\alpha-1} F_{v(1)}\right) \mid \\
\leq & I_{q}^{1-\beta_{i}}\left(I_{q}^{\alpha-1}\left|F_{u(s)}-F_{\nu(s)}\right|\right) \\
& +a_{3} I_{q}^{1-\beta_{i}}\left(I_{q}^{\alpha-1}\left|F_{u(s)}-F_{\nu(s)}\right|\right) \\
\leq & b_{1} d I_{q}^{1-\beta_{i}}\left(\int_{0}^{s}(s-q \tau)^{(\alpha-2)} \ell(\tau) d_{q} \tau\right) d_{q} s \\
& +a_{3} b_{2} d I_{q}^{1-\beta_{i}}\left(\int_{0}^{1}(1-q \tau)^{(\alpha-2)} \ell(\tau) d_{q} \tau\right) d_{q} s \\
\leq & \frac{b_{2} \ell^{*} d}{\Gamma_{q}\left(1-\beta_{i}\right)} k_{2} \int_{0}^{t}(t-q s)^{\left(-\beta_{i}\right)} s^{\alpha-\kappa-1} d_{q} s \\
& +\frac{a_{3} b_{2} \ell^{*} d}{\Gamma_{q}\left(1-\beta_{i}\right)} k_{2} \int_{0}^{t}(t-q s)^{\left(-\beta_{i}\right)} d_{q} s
\end{aligned}
$$




$$
\begin{aligned}
\leq & \frac{b_{2} \ell^{*} d}{\Gamma_{q}\left(1-\beta_{i}\right)} k_{2} \int_{0}^{1}(1-q s)^{\left(-\beta_{i}\right)} s^{\alpha-\kappa-1} d_{q} s \\
& +\frac{a_{3} b_{2} \ell^{*} d}{|1+b|) \Gamma_{q}\left(2-\beta_{i}\right)} k_{2},
\end{aligned}
$$

where $a_{3}=\frac{|b|}{|b+1|}$. Since

$$
B_{q}\left(\alpha-\kappa, 1-\beta_{i}\right)=\int_{0}^{1}(1-q s)^{\left(-\beta_{i}\right)} s^{\alpha-\kappa-1} d_{q} s=\frac{\Gamma_{q}(\alpha-\kappa) \Gamma_{q}\left(1-\beta_{i}\right)}{\Gamma_{q}\left(\alpha-\beta_{i}-\kappa+1\right)},
$$

we obtain

$$
\left|{ }^{c} D_{q}^{\beta_{i}}(T u)(t)-{ }^{c} D_{q}^{\beta_{i}}(T v)(t)\right| \leq\left[\frac{b_{2} \Gamma(\alpha-\kappa) \ell^{*}}{\Gamma_{q}\left(\alpha-\beta_{i}-\kappa+1\right)} k_{2}+\frac{a_{3} b_{2} \ell^{*}}{\Gamma_{q}\left(2-\beta_{i}\right)} k_{2}\right] d,
$$

for all $i=1,2, \ldots, n$. Hence, we get

$$
\begin{aligned}
\|T u-T v\| \leq & {\left[\frac{b_{1}(1+2|a|) \ell^{*}}{|1+a|} k_{1}+a_{2} b_{2} \ell^{*} k_{2}\right.} \\
& \left.+\sum_{i=1}^{n}\left(\frac{b_{2} \ell^{*} \Gamma_{q}(\alpha-\kappa)}{\Gamma_{q}\left(\alpha-\beta_{i}-\kappa+1\right)} k_{2}+\frac{a_{3} b_{2} \ell^{*}}{\Gamma_{q}\left(2-\beta_{i}\right)} k_{2}\right)\right] d \\
= & \Delta d .
\end{aligned}
$$

By assumption, $\Delta<1$, thus the mapping $F$ is a contraction and so by using the Banach contraction mapping principle, $F$ has a unique fixed point which is the unique solution of the problem (1). This completes the proof.

Corollary 1 Assume that there exists $M>0$ such that

$$
\left|F_{t, x_{i}, u_{i}}-F_{t, x_{i}^{\prime}, v_{i}}\right| \leq M\left[\sum_{i=1}^{3}\left|x_{i}-x_{i}^{\prime}\right|+\sum_{i=1}^{n}\left|u_{i}-v_{i}\right|\right],
$$

for each $t \in J$ and real numbers $x_{i}, x_{i}^{\prime}$ for $i=1,2,3, u_{i}, v_{i}$ for $i=1,2, \ldots, n$, where $F_{t, x_{i}, u_{i}}=$ $f\left(t, x_{1}, x_{2}, x_{3}, u_{1}, u_{2}, \ldots, u_{n}\right)$, and $F_{t, x_{i}^{\prime}, v_{i}}=f\left(t, x_{1}^{\prime}, x_{2}^{\prime}, x_{3}^{\prime}, v_{1}, v_{2}, \ldots, v_{n}\right)$. Then the problem (1) has a unique solution whenever

$$
\begin{aligned}
(1 & \left.+{ }_{0} \lambda_{1}+{ }_{0} \lambda_{2}\right)\left[\frac{[(1+2 a)(1+b)+b \alpha] M}{(1+a)(1+b) \Gamma_{q}(\alpha+1)}\right. \\
& \left.+\sum_{i=1}^{n}\left(\frac{M}{\Gamma_{q}\left(\alpha-\beta_{i}+1\right)}+\frac{b M}{(1+b) \Gamma_{q}\left(2-\beta_{i}\right) \Gamma_{q}(\alpha)}\right)\right]<1,
\end{aligned}
$$

where ${ }_{0} \lambda_{i}=\sup _{t \in J}\left|\int_{0}^{t} \gamma_{i}(t, s) d_{q} s\right|, i=1,2$.

Theorem 5 Let $f: J \times \mathbb{R}^{n+3} \rightarrow \mathbb{R}$ be a continuous function. In addition, we assume that there exist a positive constant $\kappa<\alpha-1$ and a function $\ell \in L^{\frac{1}{\kappa}}\left(J, \mathbb{R}^{+}\right)$. Then problem (1) has 
a solution whenever

$$
\left|F_{t, x_{j}, u_{i}}\right| \leq \ell(t)+\sum_{j=1}^{3} c_{j}\left|x_{j}\right|^{\nu_{j}}+\sum_{i=1}^{n} d_{i}\left|u_{i}\right|^{\eta_{i}}
$$

where $c_{j}, v_{j}$ belong to $[0, \infty),(0,1)$, respectively, for $j=1,2,3$ and $d_{i}, \eta_{i}$ belong to $[0, \infty)$, $(0,1)$, respectively, for $i=1,2, \ldots, n$, or whenever

$$
\left|F_{t, x_{j}, u_{i}}\right| \leq \sum_{i=1}^{3} c_{j}\left|x_{j}\right|^{v_{j}}+\sum_{i=1}^{n} d_{i}\left|u_{i}\right|^{\eta_{i}},
$$

where $c_{j}, v_{j}$ belong to $(0, \infty),(1, \infty)$, respectively, for $j=1,2,3$ and $d_{i}, \eta_{i}$ belong to $(0, \infty)$, $(1, \infty)$, respectively, for $i=1,2, \ldots, n$.

Proof First, we assume that the condition (7) is satisfied. Recall that $k_{1}=\left(\frac{1-\kappa}{\alpha-\kappa}\right)^{1-\kappa}$ and $k_{2}=\left(\frac{1-\kappa}{\alpha-\kappa-1}\right)^{1-\kappa}$. Let $B_{r}$ is the set of all $u \in X$ such that $\|u\|$ less than or equal to $r$; here

$$
\begin{aligned}
r \geq & \max \left\{\left((n+4) A_{0} c_{1}\right)^{\frac{1}{1-v_{1}}},\left((n+4) A_{0} c_{20} \lambda_{1}^{\nu_{2}}\right)^{\frac{1}{1-v_{2}}},\right. \\
& \left.\left((n+4) A_{0} c_{30} \lambda_{2}^{\nu_{3}}\right)^{\frac{1}{1-\nu_{3}}}, \max _{i}\left((n+4) A_{0} d_{i}\right)^{\frac{1}{1-\eta_{i}}},(n+4) K_{0}\right\}, \\
A_{0}= & \frac{(1+2|a|)[1+(1+a)|b|]}{(1+a)(1+b) \Gamma_{q}(\alpha+1)} \\
& +\sum_{i=1}^{n}\left(\frac{1}{\Gamma_{q}\left(\alpha-\beta_{i}+1\right)}+\frac{|b|}{|1+b| \Gamma_{q}(\alpha) \Gamma_{q}\left(2-\beta_{i}\right)}\right), \\
K_{0}= & \frac{(1+2|a|) \ell^{*}}{|1+a| \Gamma_{q}(\alpha)} k_{1}+\frac{|b|(1+2|a|) \ell^{*}}{|1+a||1+b| \Gamma_{q}(\alpha-1)} k_{2} \\
& +\frac{1}{\Gamma_{q}(\alpha-1)} \sum_{i=1}^{n}\left(\frac{\Gamma_{q}(\alpha-l) \ell^{*}}{\Gamma_{q}\left(\alpha-\beta_{i}-\kappa+1\right)} k_{2}+\frac{|b| \ell^{*}}{|1+b| \Gamma_{q}\left(2-\beta_{i}\right)} k_{2}\right),
\end{aligned}
$$

and $\ell^{*}=\left(\int_{0}^{1}(\ell(t))^{\frac{1}{\kappa}} d_{q} s\right)^{\kappa}$. Note that $B_{r}$ is a closed, bounded and convex subset of the Banach space $X$. For each $u \in B_{r}$, we obtain

$$
\begin{aligned}
|(T u)(t)|= & \left|I_{q}^{\alpha} F_{u(t)}-a_{0} I_{q}^{\alpha} F_{u(1)}+g(t) I_{q}^{\alpha-1} F_{u(1)}\right| \\
\leq & I_{q}^{\alpha}\left|F_{u(t)}\right|+\frac{|a|}{|1+a|} I_{q}^{\alpha}\left|F_{u(1)}\right|+\frac{|b|(1+2|a|)}{|1+a||1+b|} I_{q}^{\alpha-1}\left|F_{u(1)}\right| \\
\leq & I_{q}^{\alpha} \ell(t)+A_{r} I_{q}^{\alpha}(1)+\frac{|a|}{|1+a|} I_{q}^{\alpha} \ell(1)+\frac{|a|}{|1+a|} A_{r} I_{q}^{\alpha}(1) \\
& +\frac{|b|(1+2|a|)}{|1+a||1+b|} I_{q}^{\alpha-1} \ell(1)+\frac{|b|(1+2|a|)}{|1+a||1+b|} A_{r} I_{q}^{\alpha-1}(1) \\
\leq & \frac{1}{\Gamma_{q}(\alpha)}\left(\int_{0}^{t}\left((t-q s)^{(\alpha-1)}\right)^{\frac{1}{1-l}} d_{q} s\right)^{1-l} \\
& \times\left(\int_{0}^{t}(\ell(s))^{\frac{1}{l}} d_{q} s\right)^{l}
\end{aligned}
$$


Ntouyas and Samei Advances in Difference Equations

(2019) 2019:475

Page 10 of 20

$$
\begin{aligned}
& +\frac{|a|}{|1+a| \Gamma_{q}(\alpha)}\left(\int_{0}^{1}\left((1-q s)^{(\alpha-1)}\right)^{\frac{1}{1-l}} d_{q} s\right)^{1-l} \\
& \times\left(\int_{0}^{1}(m(s))^{\frac{1}{l}} d_{q} s\right)^{l} \\
& +\frac{|b|(1+2|a|)}{|1+a||1+b| \Gamma_{q}(\alpha-1)}\left(\int_{0}^{1}\left((1-q s)^{(\alpha-2)}\right)^{\frac{1}{1-l}} d_{q} s\right)^{1-l} \\
& \times\left(\int_{0}^{1}(m(s))^{\frac{1}{l}} d_{q} s\right)^{l} \\
& +\frac{(1+2|a|)(1+(1+\alpha)|b|)}{|1+a||1+b| \Gamma_{q}(\alpha+1)} A_{r} \\
& \leq \frac{(1+2|a|) \ell^{*}}{|1+a| \Gamma_{q}(\alpha)} k_{1}+\frac{|b|(1+2|a|) \ell^{*}}{|1+a||1+b| \Gamma_{q}(\alpha-1)} k_{2} \\
& +\frac{(1+2|a|)(1+(1+\alpha)|b|)}{|1+a||1+b| \Gamma_{q}(\alpha+1)} A_{r}
\end{aligned}
$$

where $a_{0}$ and $g(t)$ as defined in Theorem 4 (i.e. $a_{0}=\frac{a}{1+a}$ and $\left.g(t)=\frac{a b-b(1+a) t}{(1+a)(1+b)}, t \in J\right)$,

$$
F_{u(s)}=f\left(s, u(s),\left(\varphi_{1} u\right)(s),\left(\varphi_{2} u\right)(s),{ }^{c} D^{\beta_{1}} u(s),{ }^{c} D^{\beta_{2}} u(s), \ldots,{ }^{c} D^{\beta_{n}} u(s)\right)
$$

and $A_{r}=c_{1} r_{1}^{\nu}+c_{20} \lambda_{1}^{\nu_{2}} r^{\nu_{2}}+c_{30} \lambda_{2}^{\nu_{3}} r^{\nu_{3}}+\sum_{i=1}^{n} d_{i} r^{\eta_{i}}$. Also, we have

$$
\begin{aligned}
& \left|{ }^{c} D_{q}^{\beta_{i}}(T u)(t)\right|=\left|I_{q}^{1-\beta_{i}}(T u)^{\prime}(t)\right| \\
& =\left|I_{q}^{1-\beta_{i}}\left(I_{q}^{\alpha-1} F_{u(s)}-\frac{b}{1+b} I_{q}^{\alpha-1} F_{u(1)}\right)\right| \\
& \leq \int_{0}^{t} \frac{(t-q s)^{\left(-\beta_{i}\right)}}{\Gamma_{q}\left(1-\beta_{i}\right)}\left(\int_{0}^{s} \frac{(s-q \tau)^{(\alpha-2)}}{\Gamma_{q}(\alpha-1)}\left|F_{u(\tau)}\right| d_{q} \tau\right) d_{q} s \\
& +\frac{|b|}{|1+b|} \int_{0}^{t} \frac{(t-q s)^{\left(-\beta_{i}\right)}}{\Gamma_{q}\left(1-\beta_{i}\right)}\left(\int_{0}^{1} \frac{(1-q \tau)^{(\alpha-2)}}{\Gamma_{q}(\alpha-1)}\left|F_{u(\tau)}\right| d_{q} \tau\right) d_{q} s \\
& \leq \int_{0}^{t} \frac{(t-q s)^{\left(-\beta_{i}\right)}}{\Gamma_{q}\left(1-\beta_{i}\right)}\left(\int_{0}^{s} \frac{(s-q \tau)^{(\alpha-2)}}{\Gamma_{q}(\alpha-1)} \ell(\tau) d_{q} \tau\right) d_{q} s \\
& +A_{r} \int_{0}^{t} \frac{(t-q s)^{\left(-\beta_{i}\right)}}{\Gamma_{q}\left(1-\beta_{i}\right)}\left(\int_{0}^{s} \frac{(s-q \tau)^{(\alpha-2)}}{\Gamma_{q}(\alpha-1)} d_{q} \tau\right) d_{q} s \\
& +\frac{|b|}{|1+b|} \int_{0}^{t} \frac{(t-q s)^{\left(-\beta_{i}\right)}}{\Gamma_{q}\left(1-\beta_{i}\right)}\left(\int_{0}^{1} \frac{(1-q \tau)^{(\alpha-2)}}{\Gamma_{q}(\alpha-1)} \ell(\tau) d_{q} \tau\right) d_{q} s \\
& +\frac{|b|}{|1+b|} A_{r} \int_{0}^{t} \frac{(t-q s)^{\left(-\beta_{i}\right)}}{\Gamma_{q}\left(1-\beta_{i}\right)}\left(\int_{0}^{1} \frac{(1-q \tau)^{(\alpha-2)}}{\Gamma_{q}(\alpha-1)} d_{q} \tau\right) d_{q} s \\
& \leq \frac{1}{\Gamma_{q}(\alpha-1) \Gamma_{q}\left(1-\beta_{i}\right)} \int_{0}^{t}(t-q s)^{\left(-\beta_{i}\right)} \\
& \times\left[\left(\int_{0}^{s}\left((s-q \tau)^{(\alpha-2)}\right)^{\frac{1}{1-l}} d_{q} \tau\right)^{1-l}\left(\int_{0}^{s}(\ell(\tau))^{\frac{1}{l}} d_{q} \tau\right)^{l}\right] d_{q} s \\
& +\frac{A_{r}}{\Gamma_{q}(\alpha) \Gamma_{q}\left(1-\beta_{i}\right)} \int_{0}^{t}(t-q s)^{\left(-\beta_{i}\right)} s^{\alpha-1} d_{q} s
\end{aligned}
$$




$$
\begin{aligned}
& +\frac{|b|}{|1+b| \Gamma_{q}(\alpha-1) \Gamma_{q}\left(1-\beta_{i}\right)} \int_{0}^{t}(t-q s)^{\left(-\beta_{i}\right)} \\
& \times\left[\left(\int_{0}^{1}\left((1-q \tau)^{(\alpha-2)}\right)^{\frac{1}{1-l}} d_{q} \tau\right)^{1-l}\left(\int_{0}^{1}(\ell(\tau))^{\frac{1}{l}} d_{q} \tau\right)^{l}\right] d_{q} s \\
& +\frac{|b|}{|1+b| \Gamma_{q}(\alpha) \Gamma_{q}\left(2-\beta_{i}\right)} A_{r} \\
\leq & \frac{\ell^{*}}{\Gamma_{q}(\alpha-1) \Gamma_{q}\left(1-\beta_{i}\right)} k_{2} \int_{0}^{t}(t-q s)^{\left(-\beta_{i}\right)} s^{\alpha-l-1} d_{q} s \\
& +\frac{1}{\Gamma_{q}(\alpha) \Gamma_{q}\left(1-\beta_{i}\right)} A_{r} \int_{0}^{t}(t-q s)^{\left(-\beta_{i}\right)} s^{\alpha-1} d_{q} s \\
& +\frac{|b| \ell^{*}}{|1+b| \Gamma_{q}(\alpha-1) \Gamma_{q}\left(1-\beta_{i}\right)} k_{2} \int_{0}^{t}(t-q s)^{\left(-\beta_{i}\right)} d_{q} s \\
& +\frac{|b|}{|1+b| \Gamma_{q}(\alpha) \Gamma_{q}\left(2-\beta_{i}\right)} A_{r} \\
\leq & \frac{\ell^{*}}{\Gamma_{q}(\alpha-1) \Gamma_{q}\left(1-\beta_{i}\right)} k_{2} \int_{0}^{1}(1-q s)^{\left(-\beta_{i}\right)} s^{\alpha-l-1} d_{q} s \\
& +\frac{1}{\Gamma_{q}(\alpha) \Gamma_{q}\left(1-\beta_{i}\right)} A_{r} \int_{0}^{1}(1-q s)^{\left(-\beta_{i}\right)} s^{\alpha-1} d_{q} s \\
& +\frac{|b| \ell^{*}}{|1+b| \Gamma_{q}(\alpha-1) \Gamma_{q}\left(2-\beta_{i}\right)} k_{2}+\frac{|b|}{|1+b| \Gamma_{q}(\alpha) \Gamma_{q}\left(2-\beta_{i}\right)} A_{r} .
\end{aligned}
$$

Since, by considering Eq. (2),

$$
B_{q}\left(\alpha-l, 1-\beta_{i}\right)=\int_{0}^{1}(1-q s)^{\left(-\beta_{i}\right)} s^{\alpha-\kappa-1} d_{q} s=\frac{\Gamma_{q}(\alpha-l) \Gamma_{q}\left(1-\beta_{i}\right)}{\Gamma_{q}\left(\alpha-\beta_{i}-\kappa+1\right)}
$$

and on the other hand

$$
B_{q}\left(\alpha, 1-\beta_{i}\right)=\int_{0}^{1}(1-q \xi)^{\left(-\beta_{i}\right)} \xi^{\alpha-1} d_{q} \xi=\frac{\Gamma_{q}(\alpha) \Gamma_{q}\left(1-\beta_{i}\right)}{\Gamma_{q}\left(\alpha-\beta_{i}+1\right)},
$$

we conclude that

$$
\begin{aligned}
\left|{ }^{c} D_{q}^{\beta_{i}}(T u)(t)\right| \leq & \frac{\Gamma_{q}(\alpha-l) \ell^{*}}{\Gamma_{q}(\alpha-1) \Gamma_{q}\left(\alpha-\beta_{i}-\kappa+1\right)} k_{2} \\
& +\frac{|b| \ell^{*}}{|1+b| \Gamma_{q}(\alpha-1) \Gamma_{q}\left(2-\beta_{i}\right)} k_{2}+\frac{1}{\Gamma_{q}\left(\alpha-\beta_{i}+1\right)} A_{r} \\
& +\frac{|b|}{|1+b| A_{r} \Gamma_{q}(\alpha) \Gamma_{q}\left(2-\beta_{i}\right)} A_{r}
\end{aligned}
$$

for each $i=1,2, \ldots, n$. Hence,

$$
\begin{aligned}
\|T u\| \leq & \frac{(1+2|a|) \ell^{*}}{|1+a|) \Gamma_{q}(\alpha)} k_{1}+\frac{|b|(1+2|a|) \ell^{*}}{|1+a||1+b| \Gamma_{q}(\alpha-1)} k_{2} \\
& +\frac{1}{\Gamma_{q}(\alpha-1)} \sum_{i=1}^{n}\left[\frac{\Gamma_{q}(\alpha-l) \ell^{*}}{\Gamma_{q}\left(\alpha-\beta_{i}-l+1\right)} k_{2}\right.
\end{aligned}
$$




$$
\begin{aligned}
& \left.+\frac{|b| \ell^{*}}{|1+b| \Gamma_{q}(\alpha-1) \Gamma_{q}\left(2-\beta_{i}\right)} k_{2}\right] \\
& +A_{r}\left(\frac{(1+2|a|)(1+(1+\alpha)|b|)}{|1+a||1+b| \Gamma_{q}(\alpha+1)}\right. \\
& \left.+\sum_{i=1}^{n}\left[\frac{1}{\Gamma_{q}\left(\alpha-\beta_{i}+1\right)}+\frac{|b|}{|1+b| \Gamma_{q}(\alpha) \Gamma_{q}\left(2-\beta_{i}\right)}\right]\right) \\
& =K_{0}+A_{r} A_{0} \leq \frac{r}{n+4}(n+4)=r .
\end{aligned}
$$

Hence, $T$ maps $B_{r}$ into $B_{r}$. Now, suppose that $T$ satisfy the condition (8). In this case, choose

$$
\begin{aligned}
0< & r \\
\leq & \min \left\{\left(\frac{1}{(n+3) A_{0} c_{1}}\right)^{\frac{1}{v_{1}-1}},\left(\frac{1}{(n+3) A_{0} c_{20} \lambda_{1}^{\nu_{2}}}\right)^{\frac{1}{v_{2}-1}},\right. \\
& \left.\left(\frac{1}{(n+3) A_{0} c_{30} \lambda_{2}^{\nu_{2}}}\right)^{\frac{1}{v_{2}-1}}, \max _{i}\left(\frac{1}{(n+3) A_{0} d_{i}}\right)^{\frac{1}{\eta_{i}-1}}\right\} .
\end{aligned}
$$

By applying a similar argument, one can prove that $\|T u\| \leq r$ and so $T$ is a self-map on $B_{r}$. Also, one can easy to check that $T$ is continuous, because $w$ is continuous. For each $u \in B_{r}$, take

$$
N=\max _{t \in J}\left|w\left(t, u(t),\left(\varphi_{1} u\right)(t),\left(\varphi_{2} u\right)(t),{ }^{c} D_{q}^{\beta_{1}} u(t),{ }^{c} D_{q}^{\beta_{2}} u(t), \ldots,{ }^{c} D_{q}^{\beta_{n}} u(t)\right)\right|+1 .
$$

Thus, for each $0<t_{1}<t_{2}<1$, we have

$$
\begin{aligned}
\left|(T u)\left(t_{2}\right)-(T u)\left(t_{1}\right)\right|= & \left|I_{q}^{\alpha} F_{u\left(t_{2}\right)}-I_{q}^{\alpha} F_{u\left(t_{1}\right)}+\frac{b\left(t_{1}-t_{2}\right)}{1+b} I_{q}^{\alpha-1} F_{u(1)}\right| \\
\leq & \int_{0}^{t_{1}} \frac{\left(t_{2}-q s\right)^{(\alpha-1)}-\left(t_{1}-q s\right)^{(\alpha-1)}}{\Gamma_{q}(\alpha)}\left|F_{u(s)}\right| d_{q} s \\
& +\int_{t_{1}}^{t_{2}} \frac{\left(t_{2}-q s\right)^{(\alpha-1)}}{\Gamma_{q}(\alpha)}\left|F_{u(s)}\right| d_{q} s \\
& +\frac{|b|\left(t_{2}-t_{1}\right)}{|1+b|} \int_{0}^{1} \frac{(1-q s)^{(\alpha-2)}}{\Gamma_{q}(\alpha-1)}\left|F_{u(s)}\right| d_{q} s \\
\leq & N \int_{0}^{t_{1}} \frac{\left(t_{2}-q s\right)^{(\alpha-1)}-\left(t_{1}-q s\right)^{(\alpha-1)}}{\Gamma_{q}(\alpha)} d_{q} s \\
& +N \int_{t_{1}}^{t_{2}} \frac{\left(t_{2}-q s\right)^{(\alpha-1)}}{\Gamma_{q}(\alpha)} d_{q} s \\
& +\frac{N|b|\left(t_{2}-t_{1}\right)}{|1+b|} \int_{0}^{1} \frac{(1-q s)^{(\alpha-2)}}{\Gamma_{q}(\alpha-1)} d_{q} s \\
= & \frac{N}{\Gamma_{q}(\alpha+1)}\left(t_{2}^{\alpha}-t_{1}^{\alpha}\right)+\frac{N|b|}{|1+b| \Gamma_{q}(\alpha)}\left(t_{2}-t_{1}\right) .
\end{aligned}
$$


Furthermore, for all $i=1,2, \ldots, n$, we obtain

$$
\begin{aligned}
& \left|{ }^{c} D_{q}^{\beta_{i}}(T u)\left(t_{2}\right)-{ }^{c} D_{q}^{\beta_{i}}(T u)\left(t_{1}\right)\right| \\
& =\left|\int_{0}^{t_{2}} \frac{\left(t_{2}-q s\right)^{\left(-\beta_{i}\right)}}{\Gamma_{q}\left(1-\beta_{i}\right)}(T u)^{\prime}(s) d_{q} s-\int_{0}^{t_{1}} \frac{\left(t_{1}-q s\right)^{\left(-\beta_{i}\right)}}{\Gamma_{q}\left(1-\beta_{i}\right)}(T u)^{\prime}(s) d_{q} s\right| \\
& \leq \int_{0}^{t_{1}} \frac{\left(t_{1}-q s\right)^{\left(-\beta_{i}\right)}-\left(t_{2}-q s\right)^{\left(-\beta_{i}\right)}}{\Gamma_{q}\left(1-\beta_{i}\right)}\left(\int_{0}^{s} \frac{(s-q \tau)^{(\alpha-2)}}{\Gamma_{q}(\alpha-1)}\left|F_{u(\tau)}\right| d_{q} \tau\right) d_{q} s \\
& +\frac{|b|}{|1+b|} \int_{0}^{t_{1}} \frac{\left(t_{1}-q s\right)^{\left(-\beta_{i}\right)}-\left(t_{2}-q s\right)^{\left(-\beta_{i}\right)}}{\Gamma_{q}\left(1-\beta_{i}\right)} \\
& \times\left(\int_{0}^{1} \frac{(1-q \tau)^{(\alpha-2)}}{\Gamma_{q}(\alpha-1)}\left|F_{u(\tau)}\right| d_{q} \tau\right) d_{q} s \\
& +\int_{t_{1}}^{t_{2}} \frac{\left(t_{2}-q s\right)^{\left(-\beta_{i}\right)}}{\Gamma_{q}\left(1-\beta_{i}\right)}\left(\int_{0}^{s} \frac{(s-q \tau)^{(\alpha-2)}}{\Gamma_{q}(\alpha-1)}\left|F_{u(\tau)}\right| d_{q} \tau\right) d_{q} s \\
& +\frac{|b|}{|1+b|} \int_{t_{1}}^{t_{2}} \frac{\left(t_{2}-q s\right)^{\left(-\beta_{i}\right)}}{\Gamma_{q}\left(1-\beta_{i}\right)}\left(\int_{0}^{1} \frac{(1-q \tau)^{(\alpha-2)}}{\Gamma_{q}(\alpha-1)}\left|F_{u(\tau)}\right| d_{q} \tau\right) d_{q} s \\
& \leq \frac{N}{\Gamma_{q}(\alpha)} \int_{0}^{t_{1}} \frac{\left(t_{1}-q s\right)^{\left(-\beta_{i}\right)}-\left(t_{2}-q s\right)^{\left(-\beta_{i}\right)}}{\Gamma_{q}\left(1-\beta_{i}\right)} s^{\alpha-1} d_{q} s \\
& +\frac{N|b|}{|1+b| \Gamma_{q}(\alpha)} \int_{0}^{t_{1}} \frac{\left(t_{1}-q s\right)^{\left(-\beta_{i}\right)}-\left(t_{2}-q s\right)^{\left(-\beta_{i}\right)}}{\Gamma_{q}\left(1-\beta_{i}\right)} d_{q} s \\
& +\frac{N}{\Gamma_{q}(\alpha)} \int_{t_{1}}^{t_{2}} \frac{\left(t_{2}-q s\right)^{\left(-\beta_{i}\right)}}{\Gamma_{q}\left(1-\beta_{i}\right)} s^{\alpha-1} d_{q} s \\
& +\frac{N|b|}{|1+b| \Gamma_{q}(\alpha)} \int_{t_{1}}^{t_{2}} \frac{\left(t_{2}-q s\right)^{\left(-\beta_{i}\right)}}{\Gamma_{q}\left(1-\beta_{i}\right)} d_{q} s \\
& \leq \frac{(1+2|b|) N}{|1+b| \Gamma_{q}(\alpha)} \int_{0}^{t_{1}} \frac{\left(t_{1}-q s\right)^{\left(-\beta_{i}\right)}-\left(t_{2}-q s\right)^{\left(-\beta_{i}\right)}}{\Gamma_{q}\left(1-\beta_{i}\right)} d_{q} s \\
& +\frac{(1+2|b|) N}{|1+b| \Gamma_{q}(\alpha)} \int_{t_{1}}^{t_{2}} \frac{\left(t_{2}-q s\right)^{\left(-\beta_{i}\right)}}{\Gamma_{q}\left(1-\beta_{i}\right)} d_{q} s \\
& \leq \frac{(1+2|b|) N}{|1+b| \Gamma_{q}(\alpha) \Gamma_{q}\left(2-\beta_{i}\right)}\left[\left(t_{2}^{1-\beta_{i}}-t_{1}^{1-\beta_{i}}\right)+2\left(t_{2}-t_{1}\right)^{1-\beta_{i}}\right] .
\end{aligned}
$$

Hence,

$$
\begin{aligned}
\left\|T u\left(t_{2}\right)-T u\left(t_{1}\right)\right\| \leq & \frac{N}{\Gamma_{q}(\alpha+1)}\left(t_{2}^{\alpha}-t_{1}^{\alpha}\right)+\frac{N|b|}{|1+b| \Gamma_{q}(\alpha)}\left(t_{2}-t_{1}\right) \\
& +\sum_{i=1}^{n} \frac{(1+2|b|) N}{|1+b| \Gamma_{q}(\alpha) \Gamma_{q}\left(2-\beta_{i}\right)}\left[\left(t_{2}^{1-\beta_{i}}-t_{1}^{1-\beta_{i}}\right)\right. \\
& \left.+2\left(t_{2}-t_{1}\right)^{1-\beta_{i}}\right]
\end{aligned}
$$

which implies that $\left\|T u\left(t_{2}\right)-T u\left(t_{1}\right)\right\| \rightarrow 0$ as $t_{1} \rightarrow t_{2}$. Thus, $T$ is uniformly bounded and equicontinuous and so the theorem of Arzelá-Ascoli implies that $T$ is completely continuous. At present, from Theorem 1, $T$ has a fixed point in $B_{r}$. Finally, the problem (1) has a solution. 
Corollary 2 Assume that a real-valued function $f$ defined on $J \times \mathbb{R}^{n+3}$ is continuous. Then the problem (1) has at least one solution whenever there exist a positive constant $l<\alpha-1$ and a real-valued function $\ell \in L^{\frac{1}{l}}\left(J, \mathbb{R}^{+}\right)$such that $\left|w\left(t, x_{1}, x_{2}, x_{3}, u_{1}, u_{2}, \ldots, u_{n}\right)\right| \leq \ell(t)$, for each $t$ in $J$, and $x_{j}$, with $j=1,2,3$, $u_{i}$, with $1 \leq i \leq n$, in $\mathbb{R}$.

\section{Examples illustrative for the problems with algorithms}

In this part, we give complete computational techniques for checking working to illustrate of the problem (1), in our theorems, such that it covers all the problems and we present numerical examples which entail perfect solutions. Foremost, we present a simplified analysis that can be executed to calculate the value of the $q$-Gamma function, $\Gamma_{q}(x)$, for input $q, x$ and different values of $n$. To this aim, we consider a pseudo-code description of the method for calculating the $q$-Gamma function of order $n$ in Algorithm 2 (for more details, see the link https://en.wikipedia.org/wiki/Q-gamma_function). Now we give the following examples to illustrate our results.

Example 1 Consider the multi-term nonlinear fractional $q$-integro-differential equation

$$
\left\{\begin{aligned}
{ }^{c} D_{q}^{\frac{8}{5}} u(t)= & \frac{e^{-\pi t}}{30 \sqrt{\pi}+e^{-\pi t}}\left[\frac{\cos t+e^{t}}{1+t^{4}}+\frac{2|u(t)|}{1+2|u(t)|}\right. \\
& +\frac{e^{-\pi t} \sin \pi t}{1+t^{3}}\left(1+\frac{2\left|\left(\varphi_{1} u\right)(t)+{ }^{c} D_{q}^{\frac{1}{3}} u(t)\right|}{1+2\left|\left(\varphi_{1} u\right)(t)+{ }^{c} D_{q}^{\frac{1}{3}} u(t)\right|}\right) \\
& \left.+\frac{1+\cos ^{2} \pi t}{3\left(t^{\frac{4}{3}}+6\right)}\left(3\left(\varphi_{2} u\right)(t)+\frac{\left.2\right|^{c} D_{q}^{5} u(t) \mid}{1+2\left|{ }^{c} D_{q}^{5} u(t)\right|}\right)\right]
\end{aligned}\right.
$$

under boundary conditions $u(0)+u(1)=0$ and $u^{\prime}(0)+u^{\prime}(1)=0$, where $\left(\varphi_{1} u\right)(t)$ and $\left(\varphi_{2} u\right)(t)$ are defined by $\frac{1}{10} \int_{0}^{t} e^{-2(s-t)} u(s) d_{q} s$ and $\frac{1}{10} \int_{0}^{t} e^{-(s-t) / 4} u(s) d_{q} s$, respectively, with

$$
\begin{aligned}
{ }_{0} \lambda_{1} & =\sup _{t \in J}\left|\int_{0}^{t} \frac{e^{-2(s-t)}}{10} d_{q} s\right|=\sup _{t \in J}\left|t(1-q) \sum_{k=0}^{\infty} q^{k} \frac{e^{2 t\left(1-q^{k}\right)}}{10}\right| \\
& =|1-q| \sum_{k=0}^{\infty}\left|q^{k} \frac{e^{2\left(1-q^{k}\right)}}{10}\right|
\end{aligned}
$$

and

$$
\begin{aligned}
{ }_{0} \lambda_{2} & =\sup _{t \in I}\left|\int_{0}^{t} \frac{e^{-(s-t) / 4}}{10} d_{q} s\right|=\sup _{t \in I}\left|t(1-q) \sum_{k=0}^{\infty} q^{k} \frac{e^{t\left(1-q^{k}\right) / 4}}{10}\right| \\
& =|1-q| \sum_{k=0}^{\infty}\left|q^{k} \frac{e^{\left(1-q^{k}\right) / 4}}{10}\right| .
\end{aligned}
$$

Then we have

$$
\begin{aligned}
\left|F_{u(t)}-F_{v(t)}\right| \leq & \frac{1}{30 \sqrt{\pi}}(|u(t)-v(t)| \\
& +\left|\left(\varphi_{1} u\right)(t)-\left(\varphi_{1} v\right)(t)\right|+\left|\left(\varphi_{2} u\right)(t)-\left(\varphi_{2} v\right)(t)\right| \\
& \left.+\left|{ }^{c} D_{q}^{\frac{1}{3}} u(t)-{ }^{c} D_{q}^{\frac{1}{3}} v(t)\right|+\left|{ }^{c} D_{q}^{\frac{2}{5}} u(t)-{ }^{c} D_{q}^{\frac{2}{5}} v(t)\right|\right),
\end{aligned}
$$


where

$$
\begin{aligned}
& F_{u(t)}=w\left(t, u(t),\left(\varphi_{1} u\right)(t),\left(\varphi_{2} u\right)(t),{ }^{c} D_{q}^{\frac{1}{3}} u(t),{ }^{c} D_{q}^{\frac{2}{5}} u(t)\right), \\
& F_{v(t)}=w\left(t, v(t),\left(\varphi_{1} v\right)(t),\left(\varphi_{2} v\right)(t),{ }^{c} D_{q}^{\frac{1}{3}} v(t),{ }^{c} D_{q}^{\frac{2}{5}} v(t)\right) .
\end{aligned}
$$

Take $\ell(t)=\frac{1}{30 \sqrt{\pi}}$ belongs to $L^{\frac{1}{5}}\left(J, \mathbb{R}^{+}\right), \kappa=\frac{1}{5}$ and

$$
\ell^{*}=\left(\int_{0}^{1}\left(\frac{1}{30 \sqrt{\pi}}\right)^{5} d_{q} s\right)^{\frac{1}{5}}=\left((1-q) \sum_{k=0}^{\infty} \frac{q^{k}}{(30 \sqrt{\pi})^{5}}\right)^{\frac{1}{5}} .
$$

For different values of $q$, which are shown in Tables 1, 2 and 3, by using Algorithm 6, we obtain

$$
\begin{aligned}
\Delta= & \left(1+{ }_{0} \lambda_{1}+{ }_{0} \lambda_{2}\right)\left[\frac{3 \ell^{*}}{2 \Gamma_{q}(\alpha)} k_{1}+\frac{\ell^{*}}{4 \Gamma_{q}(\alpha-1)} k_{2}\right. \\
& +\frac{\Gamma_{q}(\alpha-\kappa) \ell^{*}}{\Gamma_{q}(\alpha-1)} k_{2}\left(\frac{1}{\Gamma_{q}\left(\alpha-\beta_{1}-\kappa+1\right)}+\frac{1}{\Gamma_{q}\left(\alpha-\beta_{2}-\kappa+1\right)}\right) \\
& \left.+\frac{\ell^{*}}{2 \Gamma_{q}(\alpha-1)} k_{2}\left(\frac{1}{\Gamma_{q}\left(2-\beta_{1}\right)}+\frac{1}{\Gamma_{q}\left(2-\beta_{2}\right)}\right)\right] \\
= & \left(1+{ }_{0} \lambda_{1}+{ }_{0} \lambda_{2}\right)\left[\frac{3 \ell^{*}}{2 \Gamma_{q}\left(\frac{8}{5}\right)}\left(\frac{4}{7}\right)^{\frac{4}{5}}+\frac{\ell^{*}}{4 \Gamma_{q}\left(\frac{3}{5}\right)}(2)^{\frac{4}{5}}\right. \\
& +\frac{\Gamma_{q}\left(\frac{7}{5}\right) \ell^{*}}{\Gamma_{q}\left(\frac{3}{5}\right)}(2)^{\frac{4}{5}}\left(\frac{1}{\Gamma_{q}\left(\frac{31}{15}\right)}+\frac{1}{\Gamma_{q}(2)}\right) \\
& \left.+\frac{\ell^{*}}{2 \Gamma_{q}\left(\frac{3}{5}\right)}(2)^{\frac{4}{5}}\left(\frac{1}{\Gamma_{q}\left(\frac{5}{3}\right)}+\frac{1}{\Gamma_{q}\left(\frac{8}{5}\right)}\right)\right]
\end{aligned}
$$

$<1$,

where $k_{1}=\left(\frac{1-\kappa}{\alpha-\kappa}\right)^{1-\kappa}$ and $k_{2}=\left(\frac{1-\kappa}{\alpha-\kappa-1}\right)^{1-\kappa}$. Now, by using Algorithms 1 and 2, we calculated ${ }_{0} \lambda_{1},{ }_{0} \lambda_{2}, \ell^{*}, \Gamma_{q}\left(\frac{8}{5}\right), \Gamma_{q}\left(\frac{3}{5}\right), \Gamma_{q}\left(\frac{7}{5}\right), \Gamma_{q}\left(\frac{31}{15}\right)$ and $\Gamma_{q}(2)$ for some values $n \in \mathbb{N}$ and $q \in(0,1)$.

Table 1 Some numerical results for calculation of $\Delta$ with $q=\frac{1}{3}$ and $n=15$ of Algorithm 6

\begin{tabular}{rlllll}
\hline$n$ & $0 \lambda_{1}$ & $0 \lambda_{2}$ & $\ell^{*}$ & $\Omega$ & $\Delta$ \\
\hline 1 & 0.084304 & 0.026252 & 0.013921 & 0.073438 & 0.081557 \\
2 & 0.128131 & 0.035503 & 0.014745 & 0.077555 & 0.090246 \\
3 & 0.145073 & 0.038644 & 0.014983 & 0.078740 & 0.093205 \\
4 & 0.151006 & 0.039698 & 0.015059 & 0.079118 & 0.094206 \\
5 & 0.153017 & 0.040050 & 0.015084 & 0.079242 & 0.094541 \\
6 & 0.153691 & 0.040167 & 0.015092 & 0.079282 & 0.094651 \\
7 & 0.153916 & 0.040206 & 0.015095 & 0.079297 & 0.094690 \\
8 & 0.153991 & 0.040219 & 0.015096 & 0.079302 & 0.094703 \\
9 & 0.154016 & 0.040224 & 0.015096 & 0.079302 & 0.094705 \\
10 & 0.154024 & 0.040225 & 0.015097 & 0.079307 & 0.094712 \\
11 & 0.154027 & 0.040226 & 0.015097 & 0.079307 & 0.094712 \\
12 & 0.154028 & 0.040226 & 0.015097 & 0.079307 & 0.094713 \\
13 & 0.154028 & 0.040226 & 0.015097 & 0.079307 & 0.094713 \\
14 & 0.154028 & 0.040226 & 0.015097 & 0.079307 & 0.094713 \\
15 & 0.154028 & 0.040226 & 0.015097 & 0.079307 & 0.094713 \\
\hline
\end{tabular}


Table 2 Some numerical results for calculation of $\Delta$ with $q=\frac{1}{2}$ and $n=19$ of Algorithm 6

\begin{tabular}{rlllll}
\hline$n$ & $0 \lambda_{1}$ & $0 \lambda_{2}$ & $\ell^{*}$ & $\Omega$ & $\Delta$ \\
\hline 1 & 0.067957 & 0.028329 & 0.014253 & 0.071709 & 0.078613 \\
2 & 0.123978 & 0.043407 & 0.015456 & 0.077908 & 0.090949 \\
3 & 0.159944 & 0.051185 & 0.015940 & 0.080482 & 0.097475 \\
4 & 0.180322 & 0.055135 & 0.016162 & 0.081684 & 0.100917 \\
5 & 0.191168 & 0.057126 & 0.016268 & 0.082263 & 0.102688 \\
6 & 0.196763 & 0.058125 & 0.016320 & 0.082548 & 0.103589 \\
7 & 0.199605 & 0.058626 & 0.016346 & 0.082691 & 0.104045 \\
8 & 0.201037 & 0.058876 & 0.016359 & 0.082763 & 0.104274 \\
9 & 0.201755 & 0.059002 & 0.016365 & 0.082796 & 0.104386 \\
10 & 0.202115 & 0.059064 & 0.016369 & 0.082818 & 0.104448 \\
11 & 0.202296 & 0.059096 & 0.016370 & 0.082823 & 0.104473 \\
12 & 0.202386 & 0.059111 & 0.016371 & 0.082829 & 0.104488 \\
13 & 0.202431 & 0.059119 & 0.016371 & 0.082829 & 0.104493 \\
14 & 0.202453 & 0.059123 & 0.016372 & 0.082834 & 0.104502 \\
15 & 0.202465 & 0.059125 & 0.016372 & 0.082834 & 0.104503 \\
16 & 0.202470 & 0.059126 & 0.016372 & 0.082834 & 0.104503 \\
17 & 0.202473 & 0.059126 & 0.016372 & 0.082834 & 0.104504 \\
18 & 0.202475 & 0.059127 & 0.016372 & 0.082834 & 0.104504 \\
19 & 0.202475 & 0.059127 & 0.016372 & 0.082834 & 0.104504 \\
\hline
\end{tabular}

Table 1 shows these calculated values. So, from Theorem 4, the problem (9) has a unique solution. In Tables 1, 2 and 3, we put

$$
\begin{aligned}
\Omega= & \frac{3 \ell^{*}}{2 \Gamma_{q}(\alpha)} k_{1}+\frac{\ell^{*}}{4 \Gamma_{q}(\alpha-1)} k_{2} \\
& +\frac{\Gamma_{q}(\alpha-\kappa) \ell^{*}}{\Gamma_{q}(\alpha-1)} k_{2}\left(\frac{1}{\Gamma_{q}\left(\alpha-\beta_{1}-\kappa+1\right)}+\frac{1}{\Gamma_{q}\left(\alpha-\beta_{2}-\kappa+1\right)}\right) \\
& +\frac{\ell^{*}}{2 \Gamma_{q}(\alpha-1)} k_{2}\left(\frac{1}{\Gamma_{q}\left(2-\beta_{1}\right)}+\frac{1}{\Gamma_{q}\left(2-\beta_{2}\right)}\right) .
\end{aligned}
$$

Algorithm 6 shows the technique of calculation $\Delta$ which was introduced in Eq. (6). Tables 1,2 and 3 show variables of $\Delta$ when $q=\frac{1}{3}, q=\frac{1}{2}$ and $q=\frac{4}{5}$, respectively. As it is seen, always $\Delta<1$ for all $n$ and $q \in(0,1)$. In addition, when values $q$ are close to one, $\Delta$ is obtained with more values of $n$ in comparison with other rows. It is shown by underlined rows. They have been underlined in line 10 of Table 1 , line 14 of Table 2 and line 31 of Table 3.

Example 2 Consider the multi-term nonlinear fractional $q$-integro-differential equation

$$
\left\{\begin{aligned}
{ }^{c} D_{q}^{\frac{7}{4}} u(t)= & \frac{\lambda e^{-2 \pi t}}{\sqrt{1+t^{3}}}+\frac{\sin \pi t}{\sqrt{2 \pi+|u(t)|+\left|{ }^{c} D_{q}^{\frac{1}{2}} u(t)\right|}}(u(t))^{\sigma_{1}} \\
& +\frac{e^{-2 \pi t}\left(1+\cos ^{2} u(t)\right)}{(t+6)^{2}}\left(\left(\varphi_{1} u\right)(t)\right)^{\sigma_{2}} \\
& +\frac{t u(t)}{\left(5+t^{2}\right)(1+|u(t)|)}\left(\left(\varphi_{2} u\right)(t)\right)^{\sigma_{3}} \\
& +\frac{(1+\alpha)\left(t-\frac{1}{2}\right)^{2}}{\Gamma_{q}(\alpha)\left(1+\left|u(t)+{ }^{c} D_{q}^{\frac{3}{2}} u(t)\right|\right)} \sum_{k=1}^{4}\left(\frac{\sin k \pi t}{2^{k}}\right)\left({ }^{c} D_{q}^{\beta_{k}} u(t)\right)^{\delta_{k}},
\end{aligned}\right.
$$


Table 3 Some numerical results for calculation of $\Delta$ with $q=\frac{4}{5}$ and $n=35$ of Algorithm 6

\begin{tabular}{|c|c|c|c|c|c|}
\hline$n$ & ${ }_{0} \lambda_{1}$ & ${ }_{0} \lambda_{2}$ & $\ell^{*}$ & $\Omega$ & $\Delta$ \\
\hline 1 & 0.023869 & 0.016820 & 0.013036 & 0.056019 & 0.058298 \\
\hline 2 & 0.050166 & 0.030826 & 0.014662 & 0.064329 & 0.069539 \\
\hline 3 & 0.077341 & 0.042394 & 0.015581 & 0.069665 & 0.078006 \\
\hline 4 & 0.104022 & 0.051889 & 0.016186 & 0.073504 & 0.084964 \\
\hline 5 & 0.129167 & 0.059642 & 0.016613 & 0.076388 & 0.090810 \\
\hline 6 & 0.152100 & 0.065947 & 0.016925 & 0.078595 & 0.095732 \\
\hline 7 & 0.172475 & 0.071058 & 0.017158 & 0.080303 & 0.099860 \\
\hline 8 & 0.190201 & 0.075189 & 0.017337 & 0.081647 & 0.103315 \\
\hline 9 & 0.205366 & 0.078522 & 0.017474 & 0.082698 & 0.106175 \\
\hline 10 & 0.218168 & 0.081207 & 0.017581 & 0.083531 & 0.108538 \\
\hline 11 & 0.228858 & 0.083366 & 0.017665 & 0.084192 & 0.110479 \\
\hline 12 & 0.237710 & 0.085101 & 0.017731 & 0.084716 & 0.112064 \\
\hline 13 & 0.244988 & 0.086493 & 0.017783 & 0.085133 & 0.113353 \\
\hline 14 & 0.250940 & 0.087610 & 0.017824 & 0.085464 & 0.114397 \\
\hline 15 & 0.255786 & 0.088506 & 0.017857 & 0.085730 & 0.115246 \\
\hline 16 & 0.259718 & 0.089224 & 0.017883 & 0.085941 & 0.115929 \\
\hline 17 & 0.262900 & 0.089799 & 0.017904 & 0.086111 & 0.116482 \\
\hline 18 & 0.265468 & 0.090259 & 0.017920 & 0.086243 & 0.116922 \\
\hline 19 & 0.267537 & 0.090628 & 0.017933 & 0.086350 & 0.117277 \\
\hline 20 & 0.269202 & 0.090923 & 0.017944 & 0.086438 & 0.117566 \\
\hline 21 & 0.270540 & 0.091160 & 0.017952 & 0.086505 & 0.117794 \\
\hline 22 & 0.271614 & 0.091349 & 0.017959 & 0.086561 & 0.117980 \\
\hline 23 & 0.272476 & 0.091500 & 0.017964 & 0.086603 & 0.118125 \\
\hline 24 & 0.273168 & 0.091621 & 0.017968 & 0.086637 & 0.118241 \\
\hline 25 & 0.273722 & 0.091718 & 0.017972 & 0.086668 & 0.118340 \\
\hline 26 & 0.274166 & 0.091796 & 0.017975 & 0.086692 & 0.118418 \\
\hline 27 & 0.274521 & 0.091858 & 0.017977 & 0.086709 & 0.118477 \\
\hline 28 & 0.274806 & 0.091907 & 0.017979 & 0.086724 & 0.118527 \\
\hline 29 & 0.275034 & 0.091947 & 0.017980 & 0.086734 & 0.118564 \\
\hline 30 & 0.275217 & 0.091979 & 0.017981 & 0.086743 & 0.118594 \\
\hline 31 & 0.275363 & 0.092004 & 0.017982 & 0.086751 & $\underline{0.118620}$ \\
\hline 32 & 0.275480 & 0.092025 & 0.017983 & 0.086758 & $\overline{0.118642}$ \\
\hline 33 & 0.275573 & 0.092041 & 0.017983 & 0.086760 & 0.118654 \\
\hline 34 & 0.275648 & 0.092054 & 0.017984 & 0.086766 & 0.118670 \\
\hline 35 & 0.275708 & 0.092064 & 0.017984 & 0.086767 & 0.118678 \\
\hline
\end{tabular}

under boundary conditions $u(0)+\frac{1}{4} u(1)=0$ and $u^{\prime}(0)+\frac{3}{4} u^{\prime}(1)=0$, here $\beta_{1}=\frac{1}{3}, \beta_{2}=\frac{3}{5}$, $\beta_{3}=\frac{1}{2}, \beta_{4}=\frac{1}{6}, \lambda \in[0, \infty)$,

$$
\left(\varphi_{1} u\right)(t)=\int_{0}^{t} \frac{s e^{-(s-t)} u(s)}{s^{2}+4} d_{q} s, \quad\left(\varphi_{2} u\right)(t)=\int_{0}^{t} \frac{16(t-s)^{4} u(s)}{\sqrt{1+s^{2}}} d_{q} s .
$$

Hence, we obtain

$$
\begin{aligned}
\left|F_{u(t)}\right| \leq & \ell(t)+\frac{1}{\sqrt{2 \pi}}|u(t)|^{\sigma_{1}}+\frac{1}{18}\left|\left(\varphi_{1} u\right)(t)\right|^{\sigma_{2}}+\frac{1}{5}\left|\left(\varphi_{2} u\right)(t)\right|^{\sigma_{3}} \\
& +\sum_{k=1}^{4} \frac{1+\alpha}{\Gamma_{q}(\alpha) 2^{k+2}}\left|{ }^{c} D_{q}^{\beta_{k}} u(t)\right|^{\delta_{k}}
\end{aligned}
$$

where

$$
F_{u(t)}=w\left(t, u(t),\left(\varphi_{1} u\right)(t),\left(\varphi_{2} u\right)(t),{ }^{c} D_{q}^{\beta_{1}} u(t),{ }^{c} D_{q}^{\beta_{2}} u(t),{ }^{c} D_{q}^{\beta_{3}} u(t),{ }^{c} D_{q}^{\beta_{4}} u(t)\right),
$$




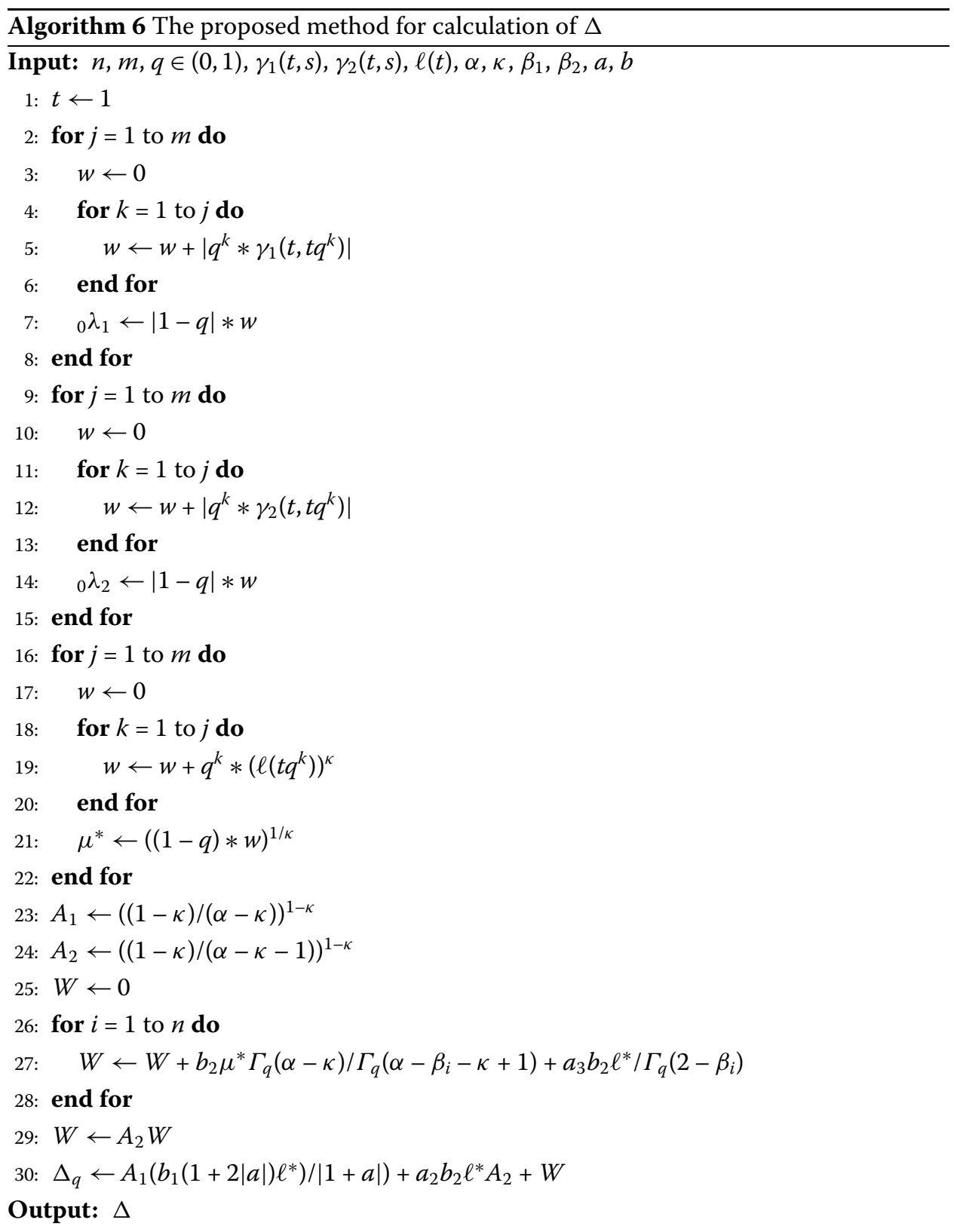

and $m(t)=\frac{\lambda e^{-\pi t}}{\sqrt{1+t^{2}}}$ for $t$ belongs to $J$. Also, if $l=\frac{1}{2}$ and $\lambda=1$, then we have

$$
\ell^{*}=\left(\int_{0}^{1}(\ell(t))^{\frac{1}{\kappa}} d_{q} s\right)^{\kappa}=\left((1-q) \sum_{k=0}^{\infty}\left(\frac{\lambda q^{k} e^{-\pi q^{k}}}{\sqrt{1+q^{2 k}}}\right)^{2}\right)^{\frac{1}{2}} .
$$

Table 4 shows the variables of $\Gamma_{q}(\alpha), \Gamma_{q}(\alpha-1), A_{0}, \ell^{*}$ and $K_{0}$ when $q=\frac{1}{3}$ and $m=1, \ldots, 40$. Since $0<\sigma_{j}$, for $j=1,2,3$, and $\delta_{i}<1$, for $i=1,2,3,4$, the assumption (7) holds. At present, if $\lambda=0, \delta_{i}>1$ and $\sigma_{j}>1$ for $i=1,2,3,4$ and $j=1,2,3$, respectively, the second condition, (8) of Theorem 5 holds. Thus, problem (10) has at least one solution. Note the features of the $q$-Gamma function, for values of $q$ close to one, the results are obtained at a greater rate of $m$. 
Table 4 Some numerical results for calculation of $\Gamma_{q}(\alpha), \Gamma_{q}(\alpha-1), A, M$ and $K$ in Theorem 5 with $q=\frac{1}{3}$ and $m=40$

\begin{tabular}{rlllll}
\hline$n$ & $\Gamma_{q}(\alpha)$ & $\Gamma_{q}(\alpha-1)$ & $A_{0}$ & $\ell^{*}$ & $K_{0}$ \\
\hline 1 & 1.054210 & 1.106942 & 6.752182 & 0.065737 & 0.174695 \\
2 & 0.996499 & 1.137694 & 6.991433 & 0.102154 & 0.271472 \\
3 & 0.970276 & 1.152228 & 7.125250 & 0.118079 & 0.313793 \\
4 & 0.957751 & 1.159303 & 7.195379 & 0.123517 & 0.328243 \\
5 & 0.951628 & 1.162796 & 7.231213 & 0.125129 & 0.332527 \\
6 & 0.948600 & 1.164531 & 7.249318 & 0.125570 & 0.33370 \\
7 & 0.947094 & 1.165395 & 7.258417 & 0.125686 & 0.334007 \\
8 & 0.946343 & 1.165827 & 7.262978 & 0.125715 & 0.334086 \\
9 & 0.945968 & 1.166043 & 7.265261 & 0.125723 & 0.334105 \\
10 & 0.945781 & 1.166150 & 7.266403 & 0.125725 & 0.334111 \\
11 & 0.945687 & 1.166204 & 7.266975 & 0.125725 & 0.334112 \\
12 & 0.945641 & 1.166231 & 7.267261 & 0.125725 & 0.334112 \\
13 & 0.945617 & 1.166245 & 7.267403 & 0.125725 & 0.334112 \\
14 & 0.945606 & 1.166251 & 7.267475 & 0.125725 & 0.334112 \\
15 & 0.945600 & 1.166255 & 7.267511 & 0.125725 & 0.334112 \\
16 & 0.945597 & 1.166256 & 7.267528 & 0.125725 & 0.334112 \\
17 & 0.945595 & 1.166257 & 7.267537 & 0.125725 & 0.334112 \\
18 & 0.945595 & 1.166258 & 7.267542 & 0.125725 & 0.334112 \\
19 & $\underline{0.945594}$ & $\underline{1.166258}$ & 7.267544 & 0.125725 & 0.334112 \\
20 & 0.945594 & 1.166258 & 7.267545 & 0.125725 & 0.334112 \\
21 & 0.945594 & 1.166258 & $\underline{7.267546}$ & 0.125725 & 0.334112 \\
22 & 0.945594 & 1.166258 & 7.267546 & 0.125725 & 0.334112 \\
23 & 0.945594 & 1.166258 & 7.267546 & 0.125725 & 0.334112 \\
24 & 0.945594 & 1.166258 & 7.267546 & 0.125725 & 0.334112 \\
\hline & & & & &
\end{tabular}

Funding

Not applicable.

Availability of data and materials

Not applicable.

Ethics approval and consent to participate

Not applicable.

\section{Competing interests}

The authors declare that they have no competing interests.

\section{Consent for publication}

Not applicable.

Authors' contributions

All authors contributed equally and significantly in this manuscript and they read and approved the final manuscript.

\section{Author details}

${ }^{1}$ Department of Mathematics, University of Ioannina, loannina, Greece. ${ }^{2}$ Nonlinear Analysis and Applied Mathematics (NAAM)-Research Group, Department of Mathematics, Faculty of Science, King Abdulaziz University, Jeddah, Saudi Arabia.

${ }^{3}$ Department of Mathematics, Faculty of Basic Science, Bu-Ali Sina University, Hamedan, Iran.

\section{Publisher's Note}

Springer Nature remains neutral with regard to jurisdictional claims in published maps and institutional affiliations.

Received: 6 October 2019 Accepted: 8 November 2019 Published online: 15 November 2019

\section{References}

1. Jackson, F.H.: q-difference equations. Am. J. Math. 32, 305-314 (1910). https://doi.org/10.2307/2370183

2. Carmichael, R.D.: The general theory of linear q-difference equations. Am. J. Math. 34, 147-168 (1912)

3. Mason, T.E.: On properties of the solution of linear $q$-difference equations with entire function coefficients. Am. J. Math. 37, 439-444 (1915)

4. Adams, C.R.: The general theory of a class of linear partial $q$-difference equations. Trans. Am. Math. Soc. 26, 283-312 (1924)

5. Adams, C.R.: Note on the integro-q-difference equations. Trans. Am. Math. Soc. 31(4), 861-867 (1929)

6. Trjitzinsky, W.J.: Analytic theory of linear q-difference equations. Acta Math. 61, 1-38 (1933). https://doi.org/10.1007/BF02547785 
7. Al-Salam, W.A.: Some fractional q-integrals and q-derivatives. Proc. Edinb. Math. Soc. 15, 135-140 (1966-1967). https://doi.org/10.1017/S0013091500011469

8. Agarwal, R.P.: Certain fractional q-integrals and q-derivatives. Proc. Camb. Philos. Soc. 66, 365-370 (1969). https://doi.org/10.1017/S0305004100045060

9. Annaby, M.H., Mansour, Z.S.: q-Fractional Calculus and Equations. Springer, Cambridge (2012). https://doi.org/10.1007/978-3-642-30898-7

10. Rajković, P.M., Marinković, S.D., Stanković, M.S.: Fractional integrals and derivatives in q-calculus. Appl. Anal. Discrete Math. 1, 311-323 (2007)

11. Stanković, M.S., Rajković, P.M., Marinković, S.D.: On q-fractional derivatives of Riemann-Liouville and Caputo type (2009). e-prints arXiv:0909.0387

12. Ahmad, B., Ntouyas, S.K., Tariboon, J.: Quantum Calculus. New Concepts, Impulsive IVPs and BVPs, Inequalities. Trends in Abstract and Applied Analysis, vol. 4. World Scientific, Hackensack (2016). https://doi.org/10.1142/10075

13. Goodrich, C., Peterson, A.: Discrete Fractional Calculus. Springer, Berlin (2015) https://doi.org/10.1007/978-3-319-25562-0

14. Ahmad, B., Ntouyas, S.K.: Existence of solutions for nonlinear fractional $q$-difference inclusions with nonlocal robin (separated) conditions. Mediterr. J. Math. 10, 133-1351 (2013). https://doi.org/10.1007/s00009-013-0258-0

15. Podlubny, I.: Fractional Differential Equations. Academic Press, San Diego (1999)

16. Hilfer, R:: Applications of Fractional Calculus in Physics. World Scientific, Singapore (2000) https://doi.org/10.1142/3779

17. Kilbas, A.A., Srivastava, H.M., Trujillo, J.J.: Theory and Applications of Fractional Differential Equations. Elsevier, Amsterdam (2006)

18. Ahmad, B., Sivasundaram, S.: On four-point nonlocal boundary value problems of nonlinear integro-differential equations of fractional order. Appl. Math. Comput. 217(2), 480-487 (2010). https://doi.org/10.1016/j.amc.2010.05.080

19. Ahmad, B., Nieto, J.J.: Riemann-Liouville fractional integro-differential equations with fractional nonlocal integral boundary conditions. Bound. Value Probl. 2011, 36 (2011). https://doi.org/10.1186/1687-2770-2011-36

20. Anguraj, A., Karthikeyan, P., Trujillo, J.J.: Existence of solutions to fractional mixed integrodifferential equations with nonlocal initial condition. Adv. Differ. Equ. 2011 (1), 690653 (2011). https://doi.org/10.1155/2011/690653

21. Rezapour, S., Shabibi, M.: A singular fractional differential equation with Riemann-Liouville integral boundary condition. J. Adv. Math. Stud. 8(1), 80-88 (2015)

22. Shabibi, M., Rezapour, S., Vaezpour, S.M.: A singular fractional integro-differential equation. UPB Sci. Bull., Ser. A 79(1) 109-118 (2017)

23. Ntouyas, S.K., Etemad, S.: On the existence of solutions for fractional differential inclusions with sum and integral boundary conditions. Appl. Math. Comput. 266, 235-243 (2017). https://doi.org/10.1016/j.amc.2015.05.036

24. Samei, M.E., Khalilzadeh Ranjbar, G.: Some theorems of existence of solutions for fractional hybrid $q$-difference inclusio. J. Adv. Math. Stud. 12(1), 63-76 (2019)

25. Samei, M.E., Ranjbar, G.K., Hedayati, V.: Existence of solutions for equations and inclusions of multi-term fractional q-integro-differential with non-separated and initial boundary conditions. J. Inequal. Appl. 2019, 273 (2019). https://doi.org/10.1186/s13660-019-2224-2

26. Ahmad, B., Ntouyas, S.K., Purnaras, I.K.: Existence results for nonlocal boundary value problems of nonlinear fractional q-difference equations. Adv. Differ. Equ. 2012, 140 (2012). https://doi.org/10.1186/1687-1847-2012-140

27. Zhao, Y., Chen, H., Zhang, Q.: Existence results for fractional $q$-difference equations with nonlocal $q$-integral boundary conditions. Adv. Differ. Equ. 2013, 48 (2013). https://doi.org/10.1186/1687-1847-2013-48

28. Ahmad, B., Nieto, J.J., Alsaedi, A., Al-Hutami, H.: Existence of solutions for nonlinear fractional q-difference integral equations with two fractional orders and nonlocal four-point boundary conditions. J. Franklin Inst. 351, 2890-2909 (2014). https://doi.org/10.1016/j.jfranklin.2014.01.020

29. Ahmad, B., Alsaedi, A., Al-Hutami, H.: A study of sequential fractional $q$-integro-difference equations with perturbed anti-periodic boundary conditions. In: Fractional Dynamics, pp. 110-128. De Gruyter, Berlin (2015) https://doi.org/10.1515/9783110472097-007

30. Ahmad, B., Ntouyas, S.K., Alsaedi, A.: Existence of solutions for fractional $q$-integro-difference inclusions with fractional q-integral boundary conditions. Adv. Differ. Equ. 2014, 257 (2014). https://doi.org/10.1186/1687-1847-2014-257

31. Ahmad, B., Etemad, S., Ettefagh, M., Rezapour, S.: On the existence of solutions for fractional $q$-difference inclusions with q-antiperiodic boundary conditions. Bull. Math. Soc. Sci. Math. Roum. 59(107)(2), 119-134 (2016)

32. Abdeljawad, T., Alzabut, J., Baleanu, D.: A generalized $q$-fractional Gronwall inequality and its applications to non-linear delay $q$-fractional difference systems. J. Inequal. Appl. 2016, 240 (2016). https://doi.org/10.1186/s13660-016-1181-2

33. Kac, V., Cheung, P.: Quantum Calculus. Universitext. Springer, New York (2002) https://doi.org/10.1007/978-1-4613-0071-7-1

34. Ferreira, R.A.C.: Nontrivial solutions for fractional $q$-difference boundary value problems. Electron. J. Qual. Theory Differ. Equ. 2010, 70 (2010)

35. Agarwal, R.P., O'Regan, D., Sahu, D.R.: Fixed Point Theory for Lipschitzian-Type Mappings with Applications. Springer, Dordrecht (2009). https://doi.org/10.1007/978-0-387-75818-3 